\title{
Characteristics of dystonic movements in primary and symptomatic dystonias
}

\author{
M Svetel, N Ivanović, J Marinković, J Jović, N Dragašević, V S Kostić
}

J Neurol Neurosurg Psychiatry 2004;75:329-330. doi: 10.1136/jnnp.2003.017632

\begin{abstract}
Objective: To compare clinical characteristics of the involuntary movements in primary and symptomatic dystonias. Patients and methods: 132 consecutive patients with the diagnosis of primary dystonia and 51 consecutive patients with secondary dystonia caused by well defined structural lesion(s) of the central nervous system, with particular emphasis on the characteristics of involuntary movements.

Results: Eight variables with the highest risk contribution to either symptomatic or primary dystonias were identified: dystonic movement in secondary dystonia was much more frequently presented at rest, whereas the presence of dystonic tremor, chronic inflammatory process, or peripheral trauma located in the region that is later affected by dystonia, as well as the use of sensory tricks and development of spontaneous remissions, classified the affected patients more often in the category of those with primary dystonia.

Conclusion: The study identified several clinical features that may be helpful in differentiating primary from secondary dystonia.
\end{abstract}

\footnotetext{
C lassification of dystonia according to aetiology differentiates between primary and secondary dystonias. ${ }^{1}$ Virtually any structural or metabolic lesions of the brain, particularly involving putamen, other basal ganglia, rostral brainstem, and upper cervical lesions, have been associated with dystonia. ${ }^{2}$ Sudden onset of occurrence at rest, cranial onset in childhood, onset in the legs during adulthood, hemidystonia, fixed postures, and early speech involvement are suggestive of secondary (symptomatic) dystonia. $^{3}$

Taking the advantage of examining a relatively large group of patients with symptomatic dystonia caused by defined structural brain lesions, ${ }^{4}$ we compared clinical characteristics of the involuntary movements in primary and symptomatic dystonias in order to define predictors for affiliation to one of these two groups.
}

\section{PATIENTS AND METHODS}

The study comprised 132 consecutive patients (52\% males) with the diagnosis of primary dystonia who were recruited for a genetic study at the Institute of Neurology (Belgrade) in the period from 1998 to 2001, and 51 consecutive patients (49\% males) with secondary dystonia caused by well defined structural lesion(s) of the central nervous system (CNS), diagnosed in the period from 1991 to 2001. The group with secondary dystonia has been previously described in detail. ${ }^{4}$ In brief, the group was composed of patients with clearly verified CNS lesion that correlated with the time of dystonia manifestation, substantiated by magnetic resonance imaging and/or computed tomography. Stroke was the main aetiology (34 patients; $66 \%$ ), followed by brain tumours (six patients; $12 \%$ ), head trauma with brain contusion (three patients; 6\%), multiple sclerosis (three patients; 6\%), arterio-venous malformations (two patients; 4\%); encephalitis (two patients; $4 \%)$, and one patient $(2 \%)$ with thalamotomy for coarse postural tremor. There were no differences between patients with primary and secondary dystonia either in age at onset (41.0 (SD 17.6) and 42.1 (SD 14.4) years, respectively), or the duration of dystonia (11.1 (SD 7.5) and 9.2 (SD 5.4) years, respectively). Positive family history for dystonia was registered in 21 patients $(16 \%)$ with primary, and in none of patients with secondary dystonia.

All the patients with primary dystonia fulfilled the following criteria: (a) presence of dystonia with different distribution (focal, segmental, multifocal, generalised); (b) normal prenatal, perinatal, and postnatal development; (c) lack of diagnostic criteria for "dystonia-plus" syndromes or other neurodegenerative diseases; (d) lack of data about toxin or drug exposure; and (e) normal neurological examination except for dystonia. Patients were observed and examined by two neurologists (VSK and MS) independently, with particular emphasis on the nature of involuntary movements.

\section{RESULTS}

Patients with primary dystonia had mainly focal dystonia (96 patients; 73\%), and less frequently segmental (26 patients; 20\%) and generalised (10 patients; $7 \%$ ) dystonia. Patients with secondary form had focal dystonia in $43 \%$ of cases (22 patients), followed by hemidystonia (16 patients; $31 \%)$, generalised ( 8 patients; $16 \%$ ), and segmental dystonia ( 5 patients; $10 \%$ ). Therefore, the appearance of focal dystonia was more frequently seen in patients with primary dystonia $(\mathrm{p}<0.001)$, while hemidystonia was present only among symptomatic cases.

Occurrence of dystonia at rest was noted in 41 (80\%) patients with secondary, and in only $55(42 \%)$ patients with primary dystonia $(p<0.001)$. The site of symptom onset did not differ in various subgroups based on dystonia distribution among patients with idiopathic and secondary forms of the disease.

Jerky clonic movements were more frequently noticed in primary cases $(p=0.001$; table 1$)$, while slow, athetoid movements were more common in symptomatic cases $(\mathrm{p}<0.001)$. Dystonic tremor of the affected body part was observed in $60 \%$ and $24 \%$ of cases with primary and secondary form of the disease, respectively $(\mathrm{p}<0.001$; table 1$)$.

Sensory tricks were used by 55 (42\%) of patients with primary, and by only two $(4 \%)$ of those with secondary dystonia $(\mathrm{p}<0.001$; table 1$)$. Complete or partial spontaneous remissions were registered in $34(26 \%)$ and two $(4 \%)$ of cases with primary and symptomatic form of the disease, respectively $(\mathrm{p}=0.001)$.

Abbreviations: CNS, central nervous system 
Table 1 Clinical characteristics of dystonic movements in patients with primary $(n=132)$ and symptomatic dystonia $(n=51)$

\begin{tabular}{llll}
\hline & $\begin{array}{l}\text { Patients with } \\
\text { symptomatic } \\
\text { dystonia }\end{array}$ & $\begin{array}{l}\text { Patients with } \\
\text { primary dystonia }\end{array}$ & p Value \\
\hline $\begin{array}{l}\text { Tonic type of dystonic } \\
\text { movement }\end{array}$ & $28 / 51(55 \%)$ & $68 / 132(52 \%)$ & 0.666 \\
Clonic (jerky) dystonic & $6 / 51(12 \%)$ & $44 / 132(33 \%)$ & $0.001^{*}$ \\
movement & & & \\
Athetoid movements & $17 / 51(33 \%)$ & $5 / 132(4 \%)$ & $0.000^{*}$ \\
$\begin{array}{l}\text { Dystonic tremor } \\
\text { Dystonia at rest/action }\end{array}$ & $12 / 51(24 \%)$ & $79 / 132(60 \%)$ & $0.000^{*}$ \\
dystonia & & $55 / 77$ & $0.000^{*}$ \\
Postural tremor & $11 / 51(22 \%)$ & $17 / 132(13 \%)$ & 0.143 \\
Other involuntary & $4 / 51(8 \%)$ & $18 / 132(14 \%)$ & 0.280 \\
movements & & & \\
Sensory tricks & $2 / 51(4 \%)$ & $56 / 132(42 \%)$ & $0.000^{*}$ \\
Spreading of dystonia & $17 / 51(33 \%)$ & $65 / 132(49 \%)$ & $0.05^{*}$ \\
Spontaneous remission & $2 / 51(4 \%)$ & $34 / 132(26 \%)$ & $0.001^{*}$ \\
Diurnal fluctuations & $12 / 51(24 \%)$ & $68 / 132(52 \%)$ & $0.001^{*}$ \\
Degree of disability & $48 / 51(94 \%)$ & $129 / 132(98 \%)$ & 0.069 \\
Pain & $32 / 51(63 \%)$ & $67 / 132(51 \%)$ & 0.145 \\
Head trauma & $2 / 51(4 \%)$ & $6 / 132(5 \%)$ & 0.853 \\
Peripheral injuries & $1 / 51(2 \%)$ & $25 / 132(19 \%)$ & $0.003^{*}$ \\
\hline * Statistically significant; values present ratio of affected v total number of \\
patients, with percentage in parenthesis.
\end{tabular}

Some form of chronic inflammatory process (such as conjunctivitis, furunculosis, phlegmona) affecting the site of dystonia presentation and antedating its occurrence was observed in 20 (15\%) patients with primary, while such cases were not found among those with secondary forms. Also, peripheral injuries preceded dystonia in $25(19 \%)$ and in only one $(2 \%)$ patients with primary and symptomatic dystonia, respectively $(\mathrm{p}=0.003$; table 1$)$.

The results of the logistical univariate regression analysis revealed differences in clinical features between these two groups. When all significant variables identified by univariate regression analysis were jointly analysed with multifactorial analysis, seven variables with the highest risk contribution to either symptomatic or primary dystonias were identified: focal distribution of dystonia (odds ratio (OR) 3.33; 95\% confidence interval (CI) 1.0 to 10.9); dystonia at rest (OR 11.96 ; $95 \%$ CI 2.8 to 51.8 ); jerky clonic movements (OR 6.42; 95\% CI 1.7 to 25.3 ); athetoid movements (OR 0.04; $95 \%$ CI 0.01 to 0.2 ); dystonic tremor (OR 5.04; $95 \%$ CI 1.4 to 18.3 ); sensory tricks (OR 24.82; 95\% CI 2.5 to 244.0); and diurnal fluctuations (OR 6.86; $95 \%$ CI 1.8 to 25.4 ).

\section{DISCUSSION}

Our study has identified several significant differences between primary and secondary dystonia: dystonic movement in secondary dystonia is much more frequently presented at rest, while the presence of dystonic tremor, chronic inflammatory process, or peripheral trauma located in the region later affected by dystonia, as well as the use of sensory tricks and development of spontaneous remissions, classified the affected patients more often in the category of those suffering from primary dystonia. Prevalence of dystonia appearance at rest suggests a secondary form of the disease. ${ }^{5}$ In both groups, focal dystonia was the most common type of dystonia, although significantly more frequently in patients with primary dystonia $(80 \% \vee 42 \% ; \mathrm{p}<0.001)$. However, hemidystonia was registered only in patients with the secondary form of the disease. This is in accordance with the data showing that at least $75 \%$ of patients with hemidystonia have a contralateral damage of the basal ganglia. ${ }^{3}$ Interestingly, contrary to suggestion that primary dystonia has gradual onset and slow progression, while symptomatic dystonia has abrupt onset followed by a rapid progression, ${ }^{6}$ we were not able to observe any difference between the groups in either the mode of onset or pace of progression.

Patients with secondary dystonia had a slightly higher incidence of dystonic posturing (that is, fixed dystonic postures), and appearance of slow, athetoid movements. However, those with primary dystonia had more frequently jerky clonic contractions. Also, dystonic tremor was observed in $60 \%$ of patients with primary and in only $24 \%$ of those with secondary dystonia.

Spontaneous remissions and the ability to suppress dystonic movement by sensory tricks also differentiate primary from secondary dystonias. Both phenomena were more frequently registered in primary dystonia, confirming the suggestion that they are typical for its clinical course. ${ }^{7}$

Data about chronic inflammatory process antedating dystonia of the same region were obtained in a survey of detailed histories in 15\% of patients with primary and in none with secondary dystonia. Similar data have been obtained for peripheral injuries. Peripheral trauma induced dystonia differs in patients with primary and those with secondary dystonia, since in the latter it appears after a short latency of only a couple of days, involuntary movements appear either at rest or during sleep, there is a slight response to anticholinergics, and there are no efficient sensory tricks. ${ }^{8}$ It was suggested that peripheral injury may cause primary dystonia particularly in genetically ${ }^{9}$ or otherwise predisposed patients. ${ }^{10}$

About $75 \%$ of all patients with dystonia suffer from primary dystonia, while secondary dystonias cover the remaining quarter of all cases. ${ }^{3}$ Our study identified several clinical features that may be helpful in differentiating primary from secondary dystonia.

\section{Authors' affiliations}

M Svetel, N Ivanović, N Dragašević, V S Kostić, Institute of Neurology CCS, School of Medicine, Belgrade, Serbia and Montenegro J Marinković, J Jović, Institute for Statistics, School of Medicine, Belgrade, Serbia and Montenegro

Correspondence to: Dr V S Kostić, Institute of Neurology CCS, UI. Dr Subotica 6, 11000 Belgrade, Yugoslavia; kostic@imi.bg.ac.yu

Received 28 April 2003

In revised form 31 May 2003

Accepted 9 June 2003

\section{REFERENCES}

1 Fahn S, Bressman S, Marsden CD. Classification of dystonia. Adv Neurol 1998; 78: 1-10

2 Kostić VS, Stojanović M, Kačar A Symptomatic dystonias associated with structural brain lesions-report of 16 cases. Can J Neurol Sci 1996;23:53-6.

3 Jankovic J, Fahn S. Dystonic disorders. In: Jankovic J, Tolosa E, eds. Parkinson's disease and movement disorders, 4th ed. Philadelphia: Lippincott Williams \& Wilkins, 2002:331-57.

4 Ivanovic $\mathbf{N}$, Svetel $M$, Kozic $D$, et al. Clinico-pathomorphological correlations in patients with symptomatic dystonias. Srp Arh Cel Lek 2002;130:183-8.

5 Tolosa ES, Martin MJ. Adult-onset idiopathic torsion dystonia. In: Wats RL, Koller CW, eds. Movement Disorders: Neurologic Principles and Practice. New York: McGraw-Hill, 1996:429-42.

6 Marsden CD. Investigation of dystonia. Adv Neurol 1998:50:35-42.

7 Lowenstein DH, Aminoff MJ. The clinical course of spasmodic torticollis. Neurology 1988;38:530-2.

8 Jankovic J. Post traumatic movement disorders: central and peripheral mechanisms. Neurology 1994:44:2006-14.

9 Turgut M, Bayulkem K, Souylu U, et al. Peripheral trauma-induced torticollis: report of four patients with normal neuroradiological studies. Mov Disord 1996;11:226.

10 Girlanda P, Quartarone A, Battaglia F, et al. Changes in spinal cord excitability in patients affected by ulnar neuropathy. Neurology 2000;55:975-8. 